\title{
Transcatheter arterial embolization for the management of persistent metrorrhagia secondary to uterine arteriovenous malformation
}

\author{
Rogerio A. Muñoz-Vigna1*, Enrique Ortiz-Herrasti², Raúl Bacelis-Arzapalo², Pedro M. Córdova-Quintal2, \\ Nora E. Lecuona-Huet ${ }^{1}$, Abraham Ziga-Martínez ${ }^{1}$ and Nicolas A. Blum-Gilbert ${ }^{1}$

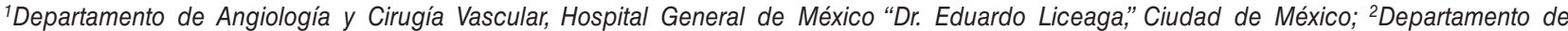 \\ Angiología y Cirugía Vascular, Hospital Regional de Alta Especialidad de la Península de Yucatán, Mérida, Yucatán. Mexico
}

\begin{abstract}
Uterine arteriovenous malformations (AVMs) are a rare pathology. They comprise a large spectrum of lesions that are classified in accordance with their content and flow. Most cases are diagnosed during an obstetric hemorrhagic event. Patients are usually asymptomatic until the occurrence of massive transvaginal bleeding. The endovascular approach is currently preferred and management will depend on the type of AVM, sclerotherapy, embolization, or surgery. We present the case of a healthy 22-year-old patient with a history of intrauterine curettage 2 months before her hospitalization due to a miscarriage. Begins with 20 days of evolution with abnormal vaginal bleeding in treatment with steroids without improvement. Ultrasound with the presence of AVM in the anterior wall of the uterus. The patient underwent endovascular embolization by transcatheter with microparticles with subsequent remission of metrorrhagia.
\end{abstract}

Key words: Uterine arteriovenous malformation. Abnormal uterine bleeding. Embolization. Microparticles.

\section{Introduction}

Uterine arteriovenous malformations (AVMs) are abnormal communications between uterine arteries and venous plexus of the myometrium. It is a rare condition, which occurs regularly in women of reproductive age and can be congenital or acquired. Acquired AVMs develop after pregnancies that do not come to term, a history of surgeries, curettage, and cesarean section with endometrial ablation or trophoblastic disease. Congenital AVMs cannot be distinguished from those acquired radiologically; thus, medical history of the patient is critically important. The most common symptom is vaginal bleeding in most cases. Diagnosis is established using ultrasound (US) initially, as well as computerized axial tomography (computed tomography [CT]) and magnetic resonance imaging (MRI) because these are not invasive methods. However, confirmation takes place by arteriography with digital subtraction. Conventionally, the definitive treatment was hysterectomy (HTA); however, the endovascular approach is currently preferred, being minimally invasive, with good results and preserving fertility ${ }^{1-3}$.

\section{Clinical case}

A 22-year-old female patient without significant chronic medical history, with a previous pregnancy that ended in miscarriage without apparent causes, underwent uterine curettage without any immediate complications.
Correspondence:

*Rogerio A. Muñoz-Vigna

E-mail: roger.med85uaslp@gmail.com

Available online: 13-09-2019

Date of reception: 21-03-2018

Date of acceptance: 03-07-2018 DOI: 10.24875/HGMX.M19000016
Rev Med Hosp Gen Mex. 2019;82(3):155-157

www.hospitalgeneral.mx 0185-1063/@ 2018 Sociedad Médica del Hospital General de Mexico. Published by Permanyer México SA de CV. This is an open access article under the CC BY-NC-ND license (http://creativecommons.org/licenses/by-nc-nd/4.0/). 


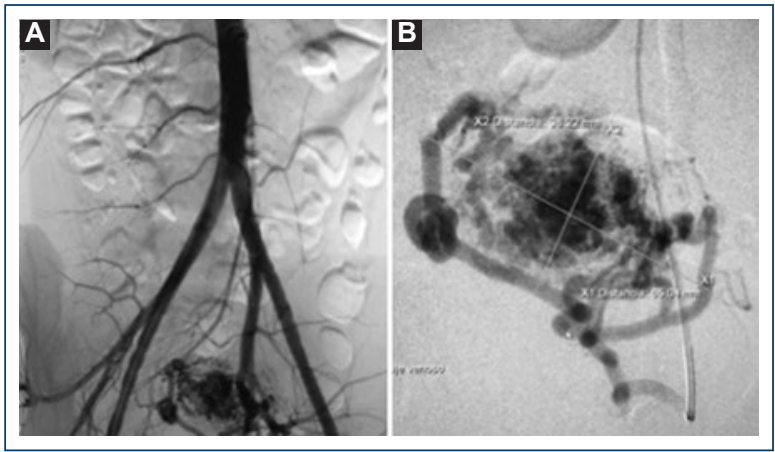

Figure 1. Initial arteriography; A: Uterine arteriovenous malformation dependent on a branch of the left uterine artery. B: Selective arteriography with the presence of malformation dependent on a single arterial branch.

The patient sought outpatient care due to a 20-day vaginal bleeding episode, with initial treatment with medroxyprogesterone without clinical improvement, decreased hemoglobin levels, and low cardiac output syndrome. Transvaginal US was performed where a heterogeneous myometrium was found, with multiple ectatic and tortuous vascular paths measuring $63 \mathrm{~mm}$ $\times 37 \mathrm{~mm} \times 55 \mathrm{~mm}$. It was complemented by a CT scan with the presence of an $82 \mathrm{~mm} \times 40 \mathrm{~mm} \times 41 \mathrm{~mm}$ uterus with a hypervascular image at the level of the anterior wall of the myometrium secondary to multiple vessels dependent on uterine arteries, with a $65 \mathrm{~mm} \times 37 \mathrm{~mm}$ "nidus." Surgical intervention was decided in the hemodynamic room. Diagnostic arteriography was performed, evidencing AVM with a dependent nidus of the left uterine artery branch measuring $65.04 \mathrm{~mm} \times 38.22 \mathrm{~mm}$ (Fig. 1), by means of selective catheterization cannulated with Envoy $^{\mathrm{TM}}$ microcatheter. An injection of polyvinyl alcohol $\left(\right.$ Contour ${ }^{\mathrm{TM}}$ ) microparticles of $355-500 \mu$ and $1 \%$ lauromacrogol foam followed in supraselective manner. Subsequently, arteriographic control revealed the absence of a contrast medium to the AVM nidus, and the procedure was completed without complications (Fig. 2). Adequate postsurgical evolution with immediate control of the haemorrhage, without complications. The patient was discharged $48 \mathrm{~h}$ after the procedure. Follow-up was performed at the outpatient unit with control CT angiography without evidence of AVM (Fig. 3).

\section{Discussion}

Uterine AVMs are an uncommon clinical entity that can jeopardize the patient's life, representing $<2 \%$ of abnormal vaginal bleeding. There are few reported cases in literature; Dubreuli and Loubat reported the first cases in 1926. It is more frequently present in

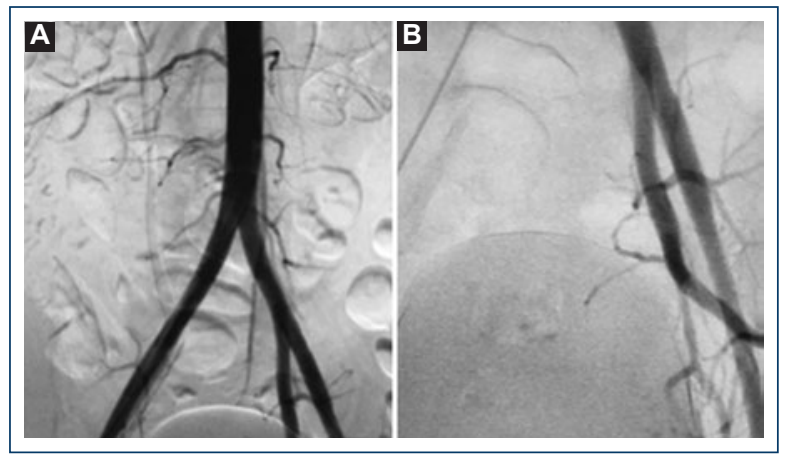

Figure 2. Control arteriography. A-B: With the absence of medium passage through AVM with total occlusion after embolization and preservation of the left hypogastric artery.

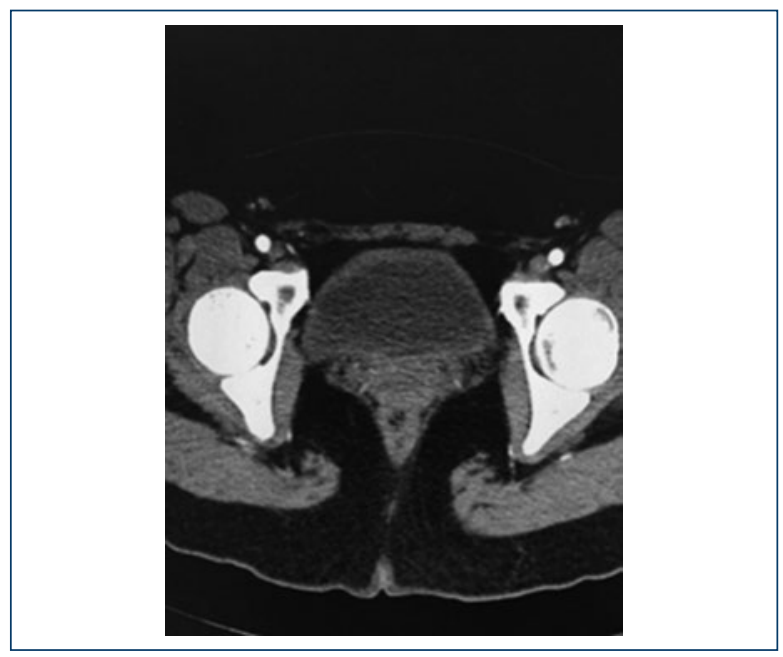

Figure 3. Control angiography at 2 months with the absence of arteriovenous malformation.

women of reproductive age and more than $95 \%$ of the patients are of premenopausal age ${ }^{1-3}$. According to Mulliken and Glowacki, they are a structural anomaly, which leads to abnormal communication between arteries and veins, are characterized by high-pressure gradients that allow high blood flow through a nidus which is composed of multiple dysplastic vascular channels that can be of low and high flow. High-flow AVMs can affect pelvic structures, visceral organs, or both. They can be congenital or acquired, in most cases, they are acquired secondary to pelvic surgery, curettage, intrauterine devices, gynecological tumors, trophoblastic disease, prolonged exposure to synthetic estrogens, or infectious processes ${ }^{2,3}$. It should be mentioned that uterine AVMs are closely related to pregnancy because the malformation appears when the venous sinuses are incorporated into the scar of the myometrium after the necrosis of the chorionic villi 
secondary to intrauterine procedures or changes related to pregnancy ${ }^{3-6}$.

Uterine AVMs are generally asymptomatically. A review made by Peitsidis et al. revealed that the most common age for presenting symptoms is 30 years. Uterine AVMs rarely affect nulliparous women, without a history of obstetric procedures or neoplastic processes. Vaginal bleeding is the most frequent symptom, often intermittent and torrential. Profuse vaginal bleeding only affects $2 \%$ of patients. Clinically, patients present with chronic anemia, sustained secondary hypotension, or even with low cardiac output syndrome. Few patients rarely experience abdominal pain, polyuria, dyspareunia, pulsatile mass, or heart failure ${ }^{2-4,7}$.

Historically, AVM diagnosis was made after HTA. At present, the trend is toward the use of less invasive studies. Transvaginal US is the first step for the diagnosis of uterine AVM. The most common echographic feature is the presence of anechoic tubular structures within the myometrium without mass effect, by means of a gray scale. In adition the spectral analysis shows, increase blood flow with high speeds, asymmetric distribution and with low resistance. CT scans or MRI provides information about the location of the lesion, related structures, and adjacent organs. Arteriography is considered the gold standard for the definitive diagnosis of AVM, in addition to offering a minimally invasive therapeutic option with preservation of fertility. In general, arteriographic findings are the presence of an opacified arterial conglomerate with an early venous filling phase. The objective is to define the vascular systems adequately and obtain a detailed anatomy of the malformation and thus be able to identify the arterial branches on which the AVM depends ${ }^{1,3,5,8}$. HTA was considered the first-choice treatment since Forssman et al. reported the first transarterial procedure of a uterine AVM. Transcatheter embolization is now considered the first treatment option. Badawy et al. reported a success rate higher than $95 \%$ in a series of 25 cases. The agents used are polyvinyl alcohol, ethyl vinyl alcohol, coils, thrombin, and sclerosing agents. The selection of material will depend on the location of the MAV, flow speed, and expertise of the surgeon. The main complications are non-selective embolization, with uterine necrosis, abscess formation, and ovarian failure. Other minor complications are hematoma and pain at the

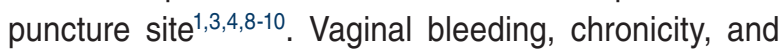
hemodynamic status of the patient should always be taken into account; in such cases, emergency surgery would be the option. Other factors to consider are the patient's age and the preservation of fertility'.

\section{Conclusions}

Uterine AVMs are a rare and dangerous clinical entity, its management is complex and a high level of suspicion is required. They occur in young women of reproductive age with a history of miscarriages, cesarean section, or uterine instrumentation. Transcatheter arterial embolization is the first, simplest, and most effective treatment with the lowest associated morbidity, reducing the rate of complications and hospital stay, mainly because it takes into account patient's needs to have their fertility preserved.

\section{Conflicts of interest}

The authors declare that there are no conflicts of interest.

\section{Ethical disclosures}

Protection of human and animal subjects. The authors declare that no experiments were performed on humans or animals for this study.

Confidentiality of data. The authors declare that they have followed the protocols of their work center on the publication of patient data.

Right to privacy and informed consent. The authors have obtained the written informed consent of the patients or subjects mentioned in the article. The corresponding author is in possession of this document.

\section{References}

1. Pankar UA, Desai $P$,Indusekhar $S$, Bhargavi V. Uterine arteriovenous malformation-beyond surgery: a case series. Int J Reprod Contracept Obstet Gynecol. 2017;6:4727-33.

2. Begum SN, Paul SN, Paul FN, Akhter P. Uterine arteriovenous malformation. A rare cause of abnormal uterine bleeding. Bangladesh J Obstet Gynaecol. 2014;29:49-53.

3. Yoon DJ, Jones M, Taani JA, Buhimschi C, Dowell JD. A systematic review of acquired uterine arteriovenous malformations: pathophysiology, diagnosis, and transcatheter treatment. Am J Perinatol Rep.2016;6:e6-14.

4. Kim SM, Ahn HY, Choi MJ, Kang YD, Park JW, Park CH, et al. Uterine arteriovenous malformation with positive serum beta-human chorionic gonadotropin: embolization of both uterine arteries and extra-uterine feeding arteries. Obstet Gynecol Sci. 2016;59:554-8.

5. Kang C, Yang S, Goo D, Kim YJ, Lee JM, Lee WH, et al. Acquired uterine vascular malformation: clinical outcome of transarterial embolization. J Korean Soc Radiol. 2017;76:104-10.

6. Christenson BM, Gipson MG, Smith MT. Pelvic vascular malformations. Semin Intervent Radiol. 2013;30:364-71.

7. Bakisololo MJ, Wang H, Mbundu MR, Linlin G. Uterine arteriovenous malformation is rare, but life-threatening disease: a report of 2 cases. Int J Cur Res Rev. 2017;9:35-39.

8. Peitsidis P, Manolakos E, Tsekoura V, Kreienberg R, Schwentner L. Uterine arteriovenous malformations induced after diagnostic curettage: a systematic review. Arch Gynecol Obstet. 2011;284:1137-51.

9. Barral PA, Saeed-Kilani M, Tradi F, Dabadie A, Izaaryene J, Soussan J, et al. Transcatheter arterial embolization with ethylene vinyl alcohol copolymer (onyx) for the treatment of hemorrhage due to uterine arteriovenous malformations. Diagn Interv Imaging. 2017;98:415-21.

10. Kim T, Shin JH, Kim J, Yoon HK, Ko GY, Gwon DI, et al. Management of bleeding uterine arteriovenous malformation with bilateral uterine artery embolization. Yonsei Med J. 2014;55:367-73. 